\title{
Frontiers in Bioengineering Research
}

Over the last 25 years, the field of bioengineering has been revolutionized in both research and education. This has been "fueled" largely by the great advances in biology and medicine over the last few decades, and the significant investments made by the Whitaker Foundation to establish new departments and more recently the Wallace H. Coulter Foundation to foster translational activities. A new engineering discipline "rooted" in biology has emerged, one which represents a transformation from a field based on the traditional engineering sciences to one that integrates engineering with biology and medicine. Bioengineering thus has become a biology-based engineering discipline with much of the research efforts hypothesis-driven and/or application-driven, achieving a quantitative, systems-level understanding of the fundamental biological behavior of living cells and animals, and the translation of advanced biotechnologies to medicine.

With this paradigm shift in bioengineering, it appeared appropriate to hold a workshop where leaders in the field could get together to discuss the state of research in key areas such as biological and medical imaging, biomaterials, and cellular and molecular bioengineering, and recommend new directions in bioengineering research over the next few decades. This workshop, sponsored by the National Science Foundation, was held February 25-26, 2013 at Georgia Institute of Technology in Atlanta. Gang Bao started the meeting with an overview of nanomedicine, with an emphasis on multifunctional nanoparticles for imaging and therapy as well as engineered nucleases for genome editing, and discussed challenges in translating nanomedicine approaches to clinical applications. What then followed was a series of sessions each focused on a specific area of bioengineering research. This special issue of Annals of Biomedical Engineering, Frontiers in Bioengineering Research, consists of 16 review articles from most of the key presenters at the workshop. As with the workshop, these reviews covered a broad range of research topics.

In the area of biological and medical imaging, Matt O'Donnell et al. described a newly developed technique, magnetomotive photoacoustic imaging, for detecting cells or molecular interactions with high sensitivity and specificity.* Roderic Pettigrew et al. summarized their

\footnotetext{
${ }^{*}$ This refers to the article available at doi:10.1007/s10439-013-0901-8.
}

recent studies on predictive biomechanical modeling of coronary plaques using an intravascular ultrasound based technique. Norbert Pelc reviewed the technology development trends in Computed Tomography since its introduction and used these trends to help illuminate likely future progress. Katherine Ferrara et al. provided a physiological perspective on the use of imaging to optimize and monitor the accumulation of nanotherapeutics within target tissues, with an emphasis on the pharmacokinetics of organic nanoparticles as drug carriers.

Concerning biomaterials and applications, David Tirrell and Kai Yuet presented recently developed tools and strategies that

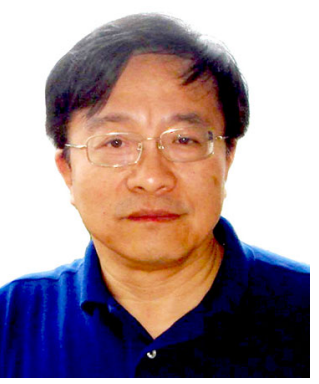

Gang Bao.

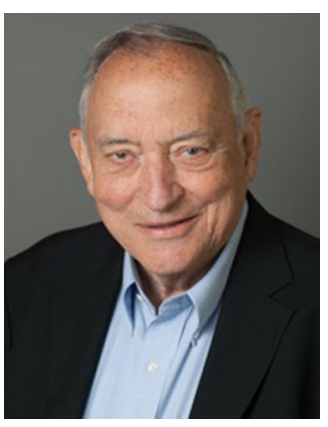

Robert M. Nerem. probe proteins synthesized during well-defined time intervals and expressed in predetermined cell types, with a focus on chemical and biological mass spectrometry-based methodologies. Andres Garcia highlighted recent progress in the engineering of poly(ethylene glycol) hydrogels cross-linked using maleimide reactive groups for protein and cell delivery. Antonios Mikos et al. presented an overview of bone and cartilage tissue engineering applications of biomaterials, which provide an architectural framework reminiscent of native extracellular matrix to encourage cell growth and eventual tissue regeneration. Ravi Bellamkonda and Nassir Mokarram reviewed the roles of different macrophages and monocytes during tissue remodeling and explored recent designs of macrophage modulatory biomaterials for tissue engineering and regenerative medicine. Todd McDevitt et al. discussed biophysical factors that influence stem cell morphogenesis, and highlighted the opportunities to employ stem cell spheroids for tissue modeling, bioprocessing, and regenerative therapies. 
There is a wide range of research activities in cellular and molecular bioengineering, as reflected by the reviews in this area. Subra Suresh and his associates provided an overview of a computational framework based on dissipative particle dynamics and its application in modeling the biorheology of whole blood and its individual components during blood flow in health and disease. Cheng Zhu summarized the recent conceptual developments of mechanical regulation of molecular interaction and the conformational change of proteins as related to mechanosensing and mechanotransduction. As a topic related to the review by Pettigrew et al., Sheldon Weinbaum examined the changing perspectives on the biomechanics of vulnerable plaque rupture over the past 25 years, especially the roles of the fibrous cap properties and the microcalcifications in the mechanism of plaque rupture. Geert Schmid-Schonbein and Marisol Chang presented an autodigestion hypothesis that degradative enzymes may play a central role in shock and multiorgan failure, and showed that blockade of digestive enzymes in the lumen of the intestine decreased the breakdown of the mucosal barrier and autodigestion of the intestine, thus reducing organ dysfunctions and mortality. Robert Guldberg et al. discussed the critical role of vascularization in normal skeletal tissue homeostasis and repair, vascular network adaptation to the local biomechanical environment, and the future directions of integrating revascularization approaches with skeletal regeneration strategies. Roger Kamm and Rashid Bashir described the opportunities and challenges in creating living machines with the potential of having self-assembly, self-repair and even self-replication abilities, and the current state-of-the-art in producing the components or building blocks of cellular machines.
The intellectual richness of the review articles in this special issue provided evidence not only on how far bioengineering research has progressed over the last 25 years, but also how this relatively young field is contributing to the understanding of biology and human physiology as well as to disease diagnosis and therapy. Due to space limitations, it is impossible to cover all the subfields of bioengineering, and some important areas of bioengineering were left out, such as bioinformatics; digital and 'wireless' medicine; medical robotics, electronics and instrumentation; and biomanufacturing. We hope that this special issue will stimulate researchers in the field to further define and develop new directions for bioengineering, ones that will likely broaden into non-medical applications such as in energy, the environment, and food, while continuing to make significant contributions to both basic and translational research for biomedical applications.

\section{GANG BAO}

Robert A. Milton Chair in Biomedical Engineering, Wallace H. Coulter Department of Biomedical Engineering, Georgia Institute of Technology and Emory University, Atlanta, GA, USA Electronic mail: gang.bao@bme.gatech.edu

\section{Robert M. Nerem}

Institute Professor Emeritus, George W. Woodruff School of Mechanical Engineering, Georgia Institute of Technology, Atlanta, GA, USA Electronic mail: robert.nerem@ibb.gatech.edu 